\title{
Synthesis of piplartine analogs and preliminary findings on structure-antimicrobial activity relationship
}

\author{
Antonio Maciel Fregnan ${ }^{1}$ - Guilherme Andrade Brancaglion ${ }^{2}$. \\ Alexandre Francisco Cerqueira Galvão ${ }^{3}$ - Cinara Oliveira D'Sousa Costa ${ }^{3}$. \\ Diogo Rodrigo Magalhães Moreira ${ }^{3,4}$ - Milena Botelho Pereira Soares ${ }^{3,4}$. \\ Daniel Pereira Bezerra ${ }^{3}$ - Naiara Chaves Silva ${ }^{5}$ - Stella Maria de Souza Morais ${ }^{5}$. \\ Josidel Conceição Oliver ${ }^{5}$ - Amanda Latercia Tranches Dias ${ }^{5} \cdot$ Luiz Felipe Leomil Coelho $^{5}$. \\ Diogo Teixeira Carvalho ${ }^{1}$. Danielle Ferreira Dias ${ }^{2} \cdot$ Thiago Belarmino de Souza ${ }^{2}$
}

Received: 23 May 2016 / Accepted: 30 December 2016 / Published online: 12 January 2017

(C) Springer Science+Business Media New York 2017

\begin{abstract}
In this work it is described the synthesis, characterization and antimicrobial and toxicity evaluation of a series of analogs of piplartine, a piperamide found in Piper sp. The compounds structures were confirmed by infrared spectroscopy, ${ }^{1} \mathrm{H},{ }^{13} \mathrm{C}$ nuclear magnetic resonance, high resolution mass spectroscopy and were evaluated against strains of Candida spp., Staphylococcus aureus, Escherichia coli and Pseudomonas aeruginosa. Derivative $\mathbf{2 4}$ was almost four-fold more potent $\left(\mathrm{IC}_{50}: 48.83 \mu \mathrm{M}\right)$ and five-fold less toxic (SI > 3) than piplartine ( IC $_{50}: 189.2 \mu \mathrm{M}$; SI: 0.21) against Candida krusei, as well as two-fold more potent than fluconazole $\left(\mathrm{IC}_{50}: 104.48 \mu \mathrm{M}\right)$. This compound was also active against Candida tropicalis at $97.67 \mu \mathrm{M}$. Benzoyl derivative 17 was three-fold more potent $\left(\mathrm{IC}_{50}: 85.2 \mu \mathrm{M}\right)$ and more than five-fold less toxic $\left(\mathrm{CC}_{50}: 231.71 \mu \mathrm{M}\right)$ than piplartine $\left(\mathrm{IC}_{50}: 315.33 \mu \mathrm{M}\right.$ and $\left.\mathrm{CC}_{50}: 41.14 \mu \mathrm{M}\right)$ against Staphylococcus aureus. Given these findings, we have
\end{abstract}

Electronic supplementary material The online version of this article (doi:10.1007/s00044-016-1774-9) contains supplementary material, which is available to authorized users.

Thiago Belarmino de Souza

thiagobs83@yahoo.com.br

1 Faculdade de Ciências Farmacêuticas, Universidade Federal de Alfenas, Alfenas 37130-000 MG, Brazil

2 Instituto de Química, Universidade Federal de Alfenas, Alfenas 37130-000 MG, Brazil

3 Centro de Pesquisas Gonçalo Moniz, Fundação Oswaldo Cruz, Rio de Janeiro 40296-710 BA, Brazil

4 Centro de Biotecnologia e Terapia Celular, Hospital São Rafael, São Marcos 41253-190 BA, Brazil

5 Instituto de Ciências Biomédicas, Universidade Federal de Alfenas, Alfenas 37130-000 MG, Brazil found analogs of piplartine which can be assumed as prototypes for the optimization and the development of new antimicrobial (compounds 24 and 17) agents.

Keywords Piplartine $\cdot$ Analogs $\cdot$ Antifungal activity Antibacterial activity

\section{Introduction}

Fungal and bacterial infections represent a serious problem of public health and, especially among immunocompromised patients related to AIDS, cancer, organ transplants, old age, and others factors. The increase in the cases of microbial resistance to available drugs has contributed to the rise in mortality rates associated with those infections. Thus, these factors justify the need for research and development of new antibacterial and antifungal agents as an alternative to improve the therapeutic arsenal. (Ling et al. 2015; Low and Rotstein 2011).

Natural products are one of the most rational sources of new drugs. They evolved naturally to perform specialized functions in plants and other organisms, so their refined structural backbones often allow the possibility for biological activity or structural modification to improve potency and pharmacokinetics (Chen et al. 2015). Many plant species produce secondary metabolites that are naturally toxic to bacteria, fungi and other parasitic organisms, as a defense response and fight for survival in competitive environments (Chin et al. 2006; Balunas and Kinglorn 2005; Paterson and Anderson 2005). Moreover, edible plant species often contain bioactive compounds, which justify the medicinal effects resulting from observations of their intake (Lampe 2003; Cragg and Newman 2005). 
As members of Piperaceae family, species of the genus Piper sp. are widely consumed around the world, especially among those populations that often use peppers in the preparation of traditional menus. Different biological activities have been reported for extracts of these species, their isolated constituents and derivatives thereof, such as anti-inflammatory (Sun et al. 2015), antiplatelet (Park et al. 2008), anti-diabetic (Rao et al. 2012), anti-microbial (Bezerra et al. 2013), anti-parasitary (Moraes et al. 2012; Cotinguiba et al. 2009), antitumor (Bezerra et al. 2006), and insecticide actions (Alécio et al. 1998). For a long time, it has been known that the main bioactive components found in these species, mainly in the roots, are alkaloid amides called piperamides (Okwute and Egharevba 2013). Piplartine, also known as piperlongumine, is an important piperamide found in Piper sp., accounting for many of the biological actions associated with the consumption of these species or their extracts.

Piplartine discretely inhibits the phytopathogenic fungus Pyricularia grisea (Lee et al. 2001), has antifungal activities against Cladosporium sphaerospermum and C. cladosporioides, comparable to nystatin and miconazole (Navickiene et al. 2006; Silva et al. 2012) and is also able to inhibit the production of mycotoxins by some species of Aspergillus and Penicillium in agricultural products (Lee et al. 2001, 2007). An antibacterial study of isolates from $P$. longum showed that piplartine was four times more potent than benzylpenicillin against Gram-positive bacteria Bacillus subtilis (Reddy et al. 2011). Another study found that piplartine could also inhibit the growth of Klebsiella pneumoniae, Pseudomonas aeruginosa and Staphylococcus aureus in a concentration-dependent manner (Naika et al. 2010). Some derivatives showed promising activities of a set of piplartine-like piperamides against $S$. aureus, B. subtilis, Aspergillus flavus and Candida albicans (Prashanth et al. 2012). Analogs of piplartine with a 4-alkoxy cinnamic moiety have exhibited submicromolar minimum inhibitory concentrations against Mycobacterium tuberculosis (Prithwiraj et al. 2011). More recently, derivatives of piplartine were synthesized and evaluated as antibacterial and cytotoxic agents (Kumar et al. 2013; Adams et al. 2012); those derivatives that possessed substituents such as phenyl, 2-nitrophenyl and 2-thiophenyl at the dihydropyridone ring showed cytostatic effects in bacteria and cancer cell lines. In another recent work, the synthesis of many structural analogs of piplartine was also described and the need for electrophilic sites in these compounds for the maintenance of this biological action was proven by cytotoxicity evaluations (Adams et al. 2012).

In view of these findings and as part of our interest in synthesizing natural analogs for bioactivity and selectivity optimization (Abrão et al. 2015; Souza et al. 2014, 2015), we report herein the preparation of new piplartine-like<smiles>COc1cc(/C=C/C(=O)N2CCC=CC2=O)cc(OC)c1OC</smiles>

Fig. 1 Chemical structure of piplartine

compounds and their antifungal, antibacterial and cytotoxic activities. (Fig. 1)

\section{Materials and methods}

\section{Chemistry}

Melting point of the compounds was determined on Microquímica MOAs 301 apparatus and was uncorrected. IR spectroscopy was performed on a Shimadzu a FTIRAffinity-1 spectrometer. ${ }^{1} \mathrm{H}-\mathrm{NMR}$ and ${ }^{13} \mathrm{C}$-NMR spectra were obtained on a Bruker AC-300 spectrometer $(300 \mathrm{MHz}$ for ${ }^{1} \mathrm{H}-\mathrm{NMR}$ and $75 \mathrm{MHz}$ for ${ }^{13} \mathrm{C}$ spectra) in deuterated chloroform, dimethylsulfoxide or methanol. Chemical shifts $(\delta)$ were reported in parts per million $(\mathrm{ppm})$ with reference to tetramethylsilane as internal standard and coupling constants $(J)$ were reported in Hertz $(\mathrm{Hz})$. The following abbreviations were used for the ${ }^{1} \mathrm{H}$ multiplicities: singlet $(s)$, doublet $(d)$ and multiplet $(m)$. High resolution mass spectra (HRMS) were acquired using a LCMS-IT-TOF mass spectrometer and the samples were solubilized in $\mathrm{MeOH}+$ $0.1 \%$ formic acid, following manual injection. Reaction courses and product mixtures were monitored by thin-layer chromatography (TLC) on commercial silica gel 60 plates. For chromatography, column grade silica gel (0.040-0.063 $\mathrm{mm}$ mesh size) was employed. The $\log P$ values of the compounds were determined by ChemBioDraw Ultra 12.0 program.

\section{General procedure for the preparation of acyl chlorides 5-8}

To a solution of $2.5 \mathrm{mmol}$ of cinnamic acid in $10 \mathrm{~mL}$ of dichloromethane were added $5 \mathrm{mmol}$ of thionyl chloride and $10 \% \mathrm{mmol}$ of $\mathrm{N}, \mathrm{N}$-dimethylformamide. After this addition, the mixture was stirred at room temperature until completion of the reaction, as noted by TLC. The crude solution of the acyl chlorides in dichloromethane were used in further steps without prior treatment or purification. 
General procedure for the preparation of hydrazides 9-12

To a solution of $12.5 \mathrm{mmol}$ of hydrazine hydrate in $10 \mathrm{~mL}$ of acetonitrile at $5{ }^{\circ} \mathrm{C}$ was added dropwise $2.5 \mathrm{mmol}$ of acid chlorides 5-8. After this addition, the mixture was allowed to warm up to room temperature until completion of the reaction, as noted by TLC. Then, $50 \mathrm{~mL}$ of water were added to the mixture and the product was extracted with ethyl acetate $(3 \times 25 \mathrm{~mL})$. The organic layer was dried by anhydrous sodium sulfate, filtered, and the solvent was removed under reduced pressure. The hydrazide derivatives were used without previous purification.

\section{General procedure for the preparation of phthalimides 13-16}

To a solution of 9-12 (1.5 mmol) in glacial acetic acid (30 $\mathrm{mL}$ ) was added $1.5 \mathrm{~mol}$ of phthalic anhydride and this mixture was heated under reflux for $3 \mathrm{~h}$. The mixture was cooled and poured into iced water and the formed solid was filtered.

(E)-N-(1,3-dioxoisoindolin-2-yl)cinnamamide (13) This compound was prepared by $1.5 \mathrm{mmol}$ of 9 and $1.5 \mathrm{mmol}$ of phthalic anhydride, according to the general procedure. The product was obtained in $90 \%$ yield as white crystals; $\mathrm{mp}$ 181-182 ${ }^{\circ} \mathrm{C}$; IR ( $\left.\overline{\mathrm{U}} / \mathrm{cm}^{-1}\right)$ : 3376, 2887, 1738, 1677, 1633, 1566, 1496, 1467. ${ }^{1} \mathrm{H}-\mathrm{NMR} \quad\left(300 \mathrm{MHz}, \mathrm{CDCl}_{3}\right) \quad \delta$ : 7.86-7.79 (4H, m, Ar-H), 7.63(1H, d, olefin, $\left.J^{3}=12.0 \mathrm{~Hz}\right)$, 7.55-7.53 (2H, m, Ar-H), 7.34-7.32 (3H, Ar-H), $6.68(1 \mathrm{H}$, d, olefin, $\left.J^{3}=12.0 \mathrm{~Hz}\right), 5.38(1 \mathrm{H}, \mathrm{s}, \mathrm{N}-\mathrm{H}) .{ }^{13} \mathrm{C}-\mathrm{NMR}(75$ $\left.\mathrm{MHz}, \mathrm{CDCl}_{3}\right) \delta: 166.1$ (1C, C-9), 165.2 (2C, C-10), 143.5 (1C, C-7); 134.7 (2C, C-13), 134.3 (1C, C-1), 130.1 (2C, C11), 130.0 (1C, C-4), 128.6 (2C, C-3/C-5), 127.8 (2C, C-2/ C-6), 123.3 (2C, C-12), 116.3 (1C, C-8). HRMS-ESI: $\mathrm{m} / \mathrm{z}$ calcd. for $\mathrm{C}_{17} \mathrm{H}_{12} \mathrm{~N}_{2} \mathrm{O}_{3}(\mathrm{M}+\mathrm{Na})^{+}$: 315.0740 ; found: 315.0564 .

(E)-N-(1,3-dioxoisoindolin-2-yl)-3-(4-methoxyphenyl) acrylamide (14) This compound was prepared by $1.5 \mathrm{mmol}$ of $\mathbf{1 0}$ and $1.5 \mathrm{mmol}$ of phthalic anhydride, according to the general procedure. The product was obtained in $85 \%$ yield as white crystals; $\mathrm{mp} 233-234^{\circ} \mathrm{C}$; IR $\left(\overline{\mathrm{U}} / \mathrm{cm}^{-1}\right)$ : 3223,3008 , 1737, 1655, 1623, 1599, 1570, 1520. ${ }^{1} \mathrm{H}-\mathrm{NMR}(300 \mathrm{MHz}$, DMSO-d $\left._{6}\right) \delta: 10.77(1 \mathrm{H}, \mathrm{s}, \mathrm{N}-\mathrm{H}), 8.00-7.92(4 \mathrm{H}, \mathrm{m}, \mathrm{Ar}-\mathrm{H})$, 7.64-7.56 $(3 \mathrm{H}, \mathrm{m}$, olefin, $\mathrm{Ar}-\mathrm{H}), 7.02\left(2 \mathrm{H}, \mathrm{d}, \mathrm{Ar}-\mathrm{H}, J^{3}=\right.$ $8,8 \mathrm{~Hz}), 6.66\left(1 \mathrm{H}, \mathrm{d}\right.$, olefin, $\left.J^{3}=12.0 \mathrm{~Hz}\right), 3.80(3 \mathrm{H}, \mathrm{s}$, methylic). ${ }^{13} \mathrm{C}-\mathrm{NMR}$ (75 MHz, DMSO-d 6 ) $\delta: 165.7$ (1C, C9), 165.0 (2C, C-10), 161.4 (1C, C-4), 142.4 (1C, C-7), 135.6 (2C, C-13); 130.1 (2C, C-11), 129.9 (2C, C-2/C-6), 127.1 (1C, C-1), 124.1 (2C, C-12), 115.4 (1C, C-8), 114.9 (2C, C-3/C-5), 55.7 (1C, $\left.\mathrm{OCH}_{3}\right)$. HRMS-ESI: $\mathrm{m} / \mathrm{z}$ calcd. for $\mathrm{C}_{18} \mathrm{H}_{14} \mathrm{~N}_{2} \mathrm{O}_{4}(\mathrm{M}+\mathrm{Na})^{+}$: 345.0846 ; found: 345.0678 .

(E)-3-(3,4-dimethoxyphenyl)-N-(1,3-dioxoisoindolin-2yl)acrylamide (15) This compound was prepared by 1.5 mmol of $\mathbf{1 1}$ and $1.5 \mathrm{mmol}$ of phthalic anhydride, according to the general procedure. The product was obtained in $70 \%$ yield as white crystals; $\mathrm{mp} 189-190^{\circ} \mathrm{C}$; IR $\left(\overline{\mathrm{U}} / \mathrm{cm}^{-1}\right): 3213$, 2980, 1739, 1654, 1619, 1596, 1581, 1512, 1467. ${ }^{1} \mathrm{H}-\mathrm{NMR}$ $\left(300 \mathrm{MHz}, \mathrm{DMSO}_{6} \mathrm{~d}_{6}\right) \delta: 10.73(1 \mathrm{H}, \mathrm{s}, \mathrm{N}-\mathrm{H}), 7.99-7.93$ $(4 \mathrm{H}, \quad \mathrm{m}, \quad \mathrm{Ar}-\mathrm{H}), \quad 7.57\left(1 \mathrm{H}, \mathrm{d}, \quad\right.$ olefin, $\left.J^{3}=12.0 \mathrm{~Hz}\right)$, 7.27-7.22 (2H, m, Ar-H), $7.02\left(1 \mathrm{H}, \mathrm{d}, \mathrm{Ar}-\mathrm{H}, J^{5}=6,2 \mathrm{~Hz}\right)$, $6.69\left(1 \mathrm{H}, \mathrm{d}\right.$, olefin, $\left.J^{3}=12.0 \mathrm{~Hz}\right), 3.81(3 \mathrm{H}, \mathrm{s}$, methylic $)$, $3.80\left(3 \mathrm{H}, \mathrm{s}\right.$, methylic). ${ }^{13} \mathrm{C}-\mathrm{NMR}\left(75 \mathrm{MHz}, \mathrm{DMSO}-\mathrm{d}_{6}\right) \delta$ : 165.3 (1C, C-9), 164.6 (2C, C-10), 150.8 (1C, C-3), 148.9 (1C, C-4), 142.3 (1C, C-7), 135.2 (2C, C-13), 129.5 (2C, C11), 126.9 (1C, C-1), 123.7 (2C, C-12), 122.1 (1C, C-6), 115.2 (1C, C-8), 111.7 (1C, C-5), 110.4 (1C, C-2), 55.5 $\left(1 \mathrm{C}, \mathrm{OCH}_{3}\right), 55.4\left(1 \mathrm{C}, \mathrm{OCH}_{3}\right)$. HRMS-ESI: $\mathrm{m} / \mathrm{z}$ calcd. for $\mathrm{C}_{19} \mathrm{H}_{16} \overline{\mathrm{N}}_{2} \mathrm{O}_{5}(\mathrm{M}+\mathrm{H})^{+}$: 353.1132 ; found: 353.0984 .

(E)-N-(1,3-dioxoisoindolin-2-yl)-3-(3,4,5-trimethoxyphenyl)acrylamide (16) This compound was prepared by $1.5 \mathrm{mmol}$ of $\mathbf{1 2}$ and $1.5 \mathrm{mmol}$ of phthalic anhydride, according to the general procedure. The product was obtained in $85 \%$ yield as white crystals; $\mathrm{mp}>230^{\circ} \mathrm{C}$; IR ( $\overline{\mathrm{U}} /$ $\mathrm{cm}^{-1}$ ): $3187,2979,1791,1682,1651,1624,1588 .{ }^{1} \mathrm{H}-$ NMR $\left(300 \mathrm{MHz}\right.$, DMSO-d $\left._{6}\right) \delta: 10.78(1 \mathrm{H}, \mathrm{s}, \mathrm{N}-\mathrm{H})$, 8.00-7.93(4H, m, Ar-H), $7.58\left(1 \mathrm{H}, \mathrm{d}\right.$, olefin, $\left.J^{3}=12.0 \mathrm{~Hz}\right)$, $7.02(2 \mathrm{H}, \mathrm{s}, \mathrm{Ar}-\mathrm{H}), 6.78\left(1 \mathrm{H}, \mathrm{d}\right.$, olefin, $\left.J^{3}=12.0 \mathrm{~Hz}\right), 3.83$ (6H, s, methylic), 3.71 (3H, s, methylic). ${ }^{13} \mathrm{C}-\mathrm{NMR}(75$ MHz, DMSO-d $_{6}$ ) $\delta: 165.6$ (1C, C-9), 164.8 (2C, C-10), 153.5 (2C, C-3/C-5), 142.3 (1C, C-7), 139.7 (1C, C-4), 135.6 (2C, C-13), 130.2 (1C, C-1), 129.9 (2C, C-11), 124.1 (2C, C-12), 117.5 (1C, C-8), 105.9 (2C, C-2/C-6), 60.5 (1C, $\left.\mathrm{OCH}_{3}\right), 56.3\left(2 \mathrm{C}, \mathrm{OCH}_{3}\right)$. HRMS-ESI: $\mathrm{m} / \mathrm{z}$ calcd. for $\mathrm{C}_{20} \mathrm{H}_{18} \mathrm{~N}_{2} \mathrm{O}_{6}(\mathrm{M}+\mathrm{Na})^{+}$: 405.1057; found: 405.0894 .

\section{General procedure for the preparation of benzoylhydrazides 17-20}

To a solution of 9-12 $(1.5 \mathrm{mmol})$ in $5 \mathrm{~mL}$ of pyridine at $5^{\circ}$ $\mathrm{C}$ was added dropwise $1.5 \mathrm{mmol}$ of benzoyl chloride and after addition, the mixture was allowed to warm up to room temperature until completion of the reaction, as reported by TLC. Then $1 \mathrm{M} \mathrm{HCl}$ was added to the mixture until $\mathrm{pH} 1$ and the precipitate formed was filtered affording the pure products.

(E)-N'-cinnamoylbenzohydrazide (17) This compound was prepared by $1.5 \mathrm{mmol}$ of 9 and $1.5 \mathrm{mmol}$ of benzoyl chloride, according to the general procedure. The product was obtained in 94\% yield as white crystals; $\mathrm{mp} 210-211^{\circ}$ C; IR ( $\left.\overline{\mathrm{U}} / \mathrm{cm}^{-1}\right)$ : 3290, 3213, 3023, 1692, 1641, 1630, 1601, $1587,1511,1487 .{ }^{1} \mathrm{H}-\mathrm{NMR}(300 \mathrm{MHz}$, DMSO-d 6 ) $\delta: 10.51$ $\left(1 \mathrm{H}, \mathrm{d}, \mathrm{N}-\mathrm{H}, J^{3}=1.5 \mathrm{~Hz}\right), 10.21\left(1 \mathrm{H}, \mathrm{d}, \mathrm{N}-\mathrm{H}, J^{3}=1.5\right.$ Hz), 7.92-7.89(2H, m, Ar-H), 7.64-7.42 (9H, m, Ar-H, olefin), $6.75\left(1 \mathrm{H}, \mathrm{d}\right.$, olefin, $\left.J^{3}=12.0 \mathrm{~Hz}\right) .{ }^{13} \mathrm{C}-\mathrm{NMR}(75$ MHz, DMSO-d 6 ) $\delta: 165.4$ (1C, C-9), 164.4 (1C, C-10), 140.3 (1C, C-7), 134.5 (1C, C-1), 132.4 (1C, C-14), 131.8 
(1C, C-11), 129.8 (1C, C-4), 129.0 (2C, C-3/C-5), 128.5 (2C, C-2/C-6), 127.7 (2C, C-13), 127.4 (2C, C-12), 119.4 (1C, C-8). HRMS-ESI: $\mathrm{m} / z$ calcd. for $\mathrm{C}_{16} \mathrm{H}_{14} \mathrm{~N}_{2} \mathrm{O}_{2}$ (M $+\mathrm{Na})^{+}: 289.0947$; found: 289.0750 .

(E)-N'-(3-(4-methoxyphenyl)acryloyl)benzohydrazide (18) This compound was prepared by $1.5 \mathrm{mmol}$ of $\mathbf{1 0}$ and $1.5 \mathrm{mmol}$ of benzoyl chloride, according to the general procedure. The product was obtained in $86 \%$ yield as white crystals; mp $182-183{ }^{\circ} \mathrm{C}$; IR $\left(\overline{\mathrm{U}} / \mathrm{cm}^{-1}\right)$ : 3203, 2999, 1693, 1642, 1627, 1601, 1577, 1508, 1479. ${ }^{1} \mathrm{H}-\mathrm{NMR}(300 \mathrm{MHz}$, DMSO-d $\left._{6}\right) \delta: 10.47\left(1 \mathrm{H}, \mathrm{d}, \mathrm{N}-\mathrm{H}, J^{3}=1.5 \mathrm{~Hz}\right), 10.10(1 \mathrm{H}$, $\left.\mathrm{d}, \mathrm{N}-\mathrm{H}, J^{3}=1.5 \mathrm{~Hz}\right), 7.90\left(2 \mathrm{H}, \mathrm{d}, \mathrm{Ar}-\mathrm{H}, J^{3}=8.5 \mathrm{~Hz}\right)$, 7.59-7.50 (6H, m, Ar-H, olefin), $7.00\left(2 \mathrm{H}, \mathrm{d}, \mathrm{Ar}-\mathrm{H}, J^{3}=\right.$ $8.5 \mathrm{~Hz}), 6.60\left(1 \mathrm{H}, \mathrm{d}\right.$, olefin, $\left.J^{3}=12.0 \mathrm{~Hz}\right), 3.80(3 \mathrm{H}, \mathrm{s}$, methylic). ${ }^{13} \mathrm{C}-\mathrm{NMR}\left(75 \mathrm{MHz}, \mathrm{DMSO}-\mathrm{d}_{6}\right) \delta: 165.4(1 \mathrm{C}, \mathrm{C}-$ 9), 164.7 (1C, C-10), 160.6 (1C, C-4), 140.0 (1C, C-7), 132.4 (1C, C-14); 131.8 (1C, C-11), 129.3 (2C, C-2/C-6), 128.4 (2C, C-13), 127.4 (2C, C-12), 127.1 (1C, C-1), 116.9 (1C, C-8), 114.4 (2C, C-3/C-5), $55.3\left(1 \mathrm{C}, \mathrm{OCH}_{3}\right)$. HRMSESI: $m / z$ calcd. for $\mathrm{C}_{17} \mathrm{H}_{16} \mathrm{~N}_{2} \mathrm{O}_{3}(\mathrm{M}+\mathrm{Na})^{+}: 319.1053$; found: 319.0862 .

(E)-N'-(3-(3,4-dimethoxyphenyl)acryloyl)benzohy-

drazide (19) This compound was prepared by $1.5 \mathrm{mmol}$ of 11 and $1.5 \mathrm{mmol}$ of benzoyl chloride, according to the general procedure. The product was obtained in $82 \%$ yield as white crystals; $\mathrm{mp} 188-189^{\circ} \mathrm{C}$; IR $\left(\overline{\mathrm{U}} / \mathrm{cm}^{-1}\right): 3292,3188$, 1667, 1642, 1622, 1564, 1513, 1467. ${ }^{1} \mathrm{H}-\mathrm{NMR}(300 \mathrm{MHz}$, DMSO-d $\left._{6}\right) \delta: 10.48\left(1 \mathrm{H}, \mathrm{d}, \mathrm{N}-\mathrm{H}, J^{3}=1.5 \mathrm{~Hz}\right), 10.06(1 \mathrm{H}$, d, N-H, $\left.J^{3}=1.5 \mathrm{~Hz}\right), 7.92-7.88(1 \mathrm{H}, \mathrm{m}, \mathrm{Ar}-\mathrm{H}), 7.58-7.45$ (3H, m, Ar-H, olefin), 7.22-7.17 (2H, m, Ar-H), 7.02-6.98 $(1 \mathrm{H}, \mathrm{m}, \mathrm{Ar}-\mathrm{H}), 6.64\left(1 \mathrm{H}, \mathrm{d}\right.$, olefin, $\left.J^{3}=12.0 \mathrm{~Hz}\right), 3.81(3 \mathrm{H}$, s, methylic), 3.79 (3H, s, methylic). ${ }^{13} \mathrm{C}-\mathrm{NMR}(75 \mathrm{MHz}$, DMSO-d $\left.\mathrm{d}_{6}\right) \delta: 165.7$ (1C, C-9), 164.8 (1C, C-10), 150.4 (1C, C-5), 148.9 (1C, C-4), 140.4 (1C, C-7), 140.1 (1C, C-1), 132.5 (1C, C-14), 131.8 (1C, C-11), 128.5 (2C, C-13), 127.4 (2C, C-12), 121.6 (1C, C-2), 117.1 (1C, C-8), 111.8 (2C, C-3), $110.3(1 \mathrm{C}, \mathrm{C}-6), 55.6\left(1 \mathrm{C}, \mathrm{OCH}_{3}\right), 55.5(1 \mathrm{C}$, $\mathrm{OCH}_{3}$ ). HRMS-ESI: $\mathrm{m} / z$ calcd. for $\mathrm{C}_{18} \mathrm{H}_{18} \overline{\mathrm{N}}_{2} \mathrm{O}_{4}(\mathrm{M}+\mathrm{Na})^{+}$: 349.1159; found: 349.1004 .

(E)- $\mathrm{N}^{\prime}$-(3-(3,4,5-trimethoxyphenyl)acryloyl)benzohydrazide (20) This compound was prepared by $1.5 \mathrm{mmol}$ of 12 and $1.5 \mathrm{mmol}$ of benzoyl chloride, according to the general procedure. The product was obtained in $87 \%$ yield as white crystals; $\mathrm{mp} 183-184^{\circ} \mathrm{C}$; IR $\left(\overline{\mathrm{U}} / \mathrm{cm}^{-1}\right)$ : 3445,3326 , 3069, 2998, 1665, 1644, 1641, 1602, 1581, 1478. ${ }^{1} \mathrm{H}-\mathrm{NMR}$ $\left(300 \mathrm{MHz}\right.$, DMSO-d $\left.\mathrm{d}_{6}\right) \delta: 10.50\left(1 \mathrm{H}, \mathrm{d}, \mathrm{N}-\mathrm{H}, J^{3}=1.5 \mathrm{~Hz}\right)$, $10.10\left(1 \mathrm{H}, \mathrm{d}, \mathrm{N}-\mathrm{H}, J^{3}=1.5 \mathrm{~Hz}\right), 7.92-7.89(2 \mathrm{H}, \mathrm{m}, \mathrm{Ar}-\mathrm{H})$, 7.54-7.49 (4H, m, Ar-H, olefin), 6.69 (2H, s, Ar-H), 6.60 $\left(1 \mathrm{H}, \mathrm{d}\right.$, olefin, $\left.J^{3}=12.0 \mathrm{~Hz}\right), 3.83(6 \mathrm{H}, \mathrm{s}$, methylic), 3.69 (3H, s, methylic). ${ }^{13} \mathrm{C}-\mathrm{NMR}$ (75 MHz, DMSO-d $\left.\mathrm{d}_{6}\right) \delta: 165.3$ (1C, C-9), 164.5 (1C, C-10), 153.1 (2C, C-3/C-5), 140.4 (1C, C-7), 138.9 (1C, C-4); 132.4 (1C, C-14), 131.8 (1C, C11), 130.2 (1C, C-1), 128.4 (2C, C-13), 127.4 (2C, C-12),
118.8 (1C, C-8), 105.2 (2C, C-2/C-6), $60.1\left(1 \mathrm{C}, \mathrm{OCH}_{3}\right)$, $55.9\left(2 \mathrm{C}, \mathrm{OCH}_{3}\right)$. HRMS-ESI: $\mathrm{m} / z$ calcd. for $\mathrm{C}_{19} \mathrm{H}_{20} \overline{\mathrm{N}}_{2} \mathrm{O}_{5}$ $(\mathrm{M}+\mathrm{Na})^{+}:$379.1264; found: 379.1096 .

\section{General procedure for the preparation of amides 21-24}

To a solution of $5.6 \mathrm{mmol}$ of morpholine in $10 \mathrm{~mL}$ of dichloromethane at $5{ }^{\circ} \mathrm{C}$ was added dropwise $1.4 \mathrm{mmol}$ of acid chlorides 5-8 and after the addition, the mixture was allowed to warm up to room temperature until completion of the reaction, as noticed by TLC. Then, $50 \mathrm{~mL}$ de water were added to the mixture and the product was extracted with dichloromethane $(3 \times 25 \mathrm{~mL})$. The organic layer was dried by anhydrous sodium sulfate, filtered and the solvent was removed under reduced pressure, affording the pure amides.

(E)-1-morpholino-3-phenylprop-2-en-1-one (21) This compound was prepared by $1.4 \mathrm{mmol}$ of 5 and $5.6 \mathrm{mmol}$ of morpholine, according to the general procedure. The product was obtained in 72\% yield as white crystals; mp 90-91 ${ }^{\circ} \mathrm{C}$; IR $\left(\overline{\mathrm{U}} / \mathrm{cm}^{-1}\right)$ : 3080, 3026, 2964, 2922, 2856, 1644, 1595, 1577, 1494. ${ }^{1} \mathrm{H}-\mathrm{NMR}\left(300 \mathrm{MHz}, \mathrm{CDCl}_{3}\right) \delta: 7.69$ $\left(1 \mathrm{H}, \mathrm{d}\right.$, olefin, $\left.J^{3}=12.0 \mathrm{~Hz}\right), 7.53-7.33(5 \mathrm{H}, \mathrm{m}, \mathrm{Ar}-\mathrm{H}), 6.84$ $\left(1 \mathrm{H}, \mathrm{d}\right.$, olefin, $\left.J^{3}=12.0 \mathrm{~Hz}\right), 3.71\left(8 \mathrm{H}, \mathrm{s}\right.$, methylenic). ${ }^{13} \mathrm{C}$ NMR $\left(75 \mathrm{MHz}, \mathrm{CDCl}_{3}\right) \delta: 165.5$ (1C, C-9), 143.1 (1C, C7), 135.0 (1C, C-1), 129.7 (1C, C-4), 128.7 (2C, C-3/C-5), 127.7 (2C, C-2/C-6), 116.5 (1C, C-8), 66.8 (4C, C-10, C11). HRMS-ESI: $\mathrm{m} / z$ calcd. for $\mathrm{C}_{13} \mathrm{H}_{15} \mathrm{NO}_{2}(\mathrm{M}+\mathrm{Na})^{+}$: 240.0995 found: 240.0846 .

(E)-3-(4-methoxyphenyl)-1-morpholinoprop-2-en-1-one (22) This compound was prepared by $1.4 \mathrm{mmol}$ of $\mathbf{6}$ and $5.6 \mathrm{mmol}$ of morpholine, according to the general procedure. The product was obtained in $75 \%$ yield as white crystals; mp $105-106^{\circ} \mathrm{C}$; IR $\left(\overline{\mathrm{U}} / \mathrm{cm}^{-1}\right): 2963,2909,2860$, $1644,1593,1573,1510 .{ }^{1} \mathrm{H}-\mathrm{NMR}\left(300 \mathrm{MHz}, \mathrm{CDCl}_{3}\right) \delta$ : $7.65\left(1 \mathrm{H}, \mathrm{d}\right.$, olefin, $\left.J^{3}=12.0 \mathrm{~Hz}\right), 7.46\left(2 \mathrm{H}, \mathrm{dd}, \mathrm{Ar}-\mathrm{H}, J^{3}=\right.$ $\left.8,50 J^{4}=4,7 \mathrm{~Hz}\right), 6.88\left(2 \mathrm{H}, \mathrm{dd}, \mathrm{Ar}-\mathrm{H}, J^{3}=8,50 J^{4}=4,7\right.$ $\mathrm{Hz}), 6.70\left(1 \mathrm{H}, \mathrm{d}\right.$, olefin, $\left.J^{3}=12.0 \mathrm{~Hz}\right), 3.82(3 \mathrm{H}, \mathrm{s}$, methylic), 3.70 (8H, s, methylenic). ${ }^{13} \mathrm{C}-\mathrm{NMR}(75 \mathrm{MHz}$, $\left.\mathrm{CDCl}_{3}\right) \delta: 165.8$ (1C, C-9), 160.9 (1C, C-4), 142.9 (1C, C7), 129.3 (2C, C-2/C-6), 127.8 (1C, C-1), 114.2 (2C, C-3/C5), 113.9 (1C, C-8), 66.8 (4C, C-10, C-11), 55.3 (1C, $\left.\mathrm{OCH}_{3}\right)$, HRMS-ESI: $m / z$ calcd. for $\mathrm{C}_{14} \mathrm{H}_{17} \mathrm{NO}_{3}(\mathrm{M}+\mathrm{Na})^{+}$: 270.1101 found: 270.0921 .

(E)-3-(3,4-dimethoxyphenyl)-1-morpholinoprop-2-en-1one (23) This compound was prepared by $1.4 \mathrm{mmol}$ of 7 and $5.6 \mathrm{mmol}$ of morpholine, according to the general procedure. The product was obtained in $72 \%$ yield as white crystals; mp $120-121^{\circ} \mathrm{C}$; IR $\left(\overline{\mathrm{U}} / \mathrm{cm}^{-1}\right): 2980,2937,2857$, 1643, 1591, 1514, 1455. ${ }^{1} \mathrm{H}-\mathrm{NMR}\left(300 \mathrm{MHz}, \mathrm{CDCl}_{3}\right) \delta$ : $7.63\left(1 \mathrm{H}, \mathrm{d}\right.$, olefin, $\left.J^{3}=12.0 \mathrm{~Hz}\right), 7.10\left(1 \mathrm{H}, \mathrm{dd}, \mathrm{Ar}-\mathrm{H}, J^{3}=\right.$ $\left.8,50 \mathrm{~Hz}, J^{4}=2,0 \mathrm{~Hz}\right), 7.02\left(1 \mathrm{H}, \mathrm{d}, \mathrm{Ar}-\mathrm{H}, J^{4}=2,0 \mathrm{~Hz}\right), 6.84$ $\left(1 \mathrm{H}, \mathrm{d}, \mathrm{Ar}-\mathrm{H}, J^{3}=8.5 \mathrm{~Hz}\right), 6.68\left(1 \mathrm{H}, \mathrm{d}\right.$, olefin, $J^{3}=12.0$ 
$\mathrm{Hz}), 3.90\left(6 \mathrm{H}, \mathrm{s}\right.$, methylic), $3.70\left(8 \mathrm{H}, \mathrm{s}\right.$, methylenic). ${ }^{13} \mathrm{C}-$ NMR $\left(75 \mathrm{MHz}, \mathrm{CDCl}_{3}\right) \delta: 165.7$ (1C, C-9), 150.6 (1C, C3), 149.1 (1C, C-4), 143.2 (1C, C-7), 128.0 (1C, C-1), 121.8 (1C, C-6), 114.1 (1C, C-8), 111.0 (1C, C-5), 109.8 (1C, C2), $66.8(4 \mathrm{C}, \mathrm{C}-10, \mathrm{C}-11), 55.9\left(2 \mathrm{C}, \mathrm{OCH}_{3}\right)$. HRMS-ESI: $\mathrm{m} / \mathrm{z}$ calcd. for $\mathrm{C}_{15} \mathrm{H}_{19} \mathrm{NO}_{4}(\mathrm{M}+\mathrm{H})^{+}:-278.1387$ found: 278.1215 .

(E)-1-morpholino-3-(3,4,5-trimethoxyphenyl)prop-2-en1-one (24) This compound was prepared by $1.4 \mathrm{mmol}$ of $\mathbf{8}$ and $5.6 \mathrm{mmol}$ of morpholine, according to the general procedure. The product was obtained in $86 \%$ yield as white crystals; mp $127-128^{\circ} \mathrm{C}$; IR $\left(\overline{\mathrm{U}} / \mathrm{cm}^{-1}\right): 2951,2896,2846$, 1640, 1580, 1504, 1498. ${ }^{1} \mathrm{H}-\mathrm{NMR}\left(300 \mathrm{MHz}, \mathrm{CDCl}_{3}\right) \delta$ : $7.59\left(1 \mathrm{H}, \mathrm{d}\right.$, olefin, $\left.J^{3}=12.0 \mathrm{~Hz}\right), 6.72-6.67(3 \mathrm{H}, \mathrm{m}, \mathrm{Ar}-\mathrm{H}$, olefin), $3.87(9 \mathrm{H}, \mathrm{s}$, methylic), $3.70(8 \mathrm{H}, \mathrm{s}$, methylenic). ${ }^{13} \mathrm{C}-\mathrm{NMR}\left(75 \mathrm{MHz}, \mathrm{CDCl}_{3}\right) \delta: 165.5$ (1C, C-9), 153.4 (2C, C-3/C-5), 143.3 (1C, C-7), 139.7 (1C, C-4), 130.6 (1C, C1), 115.6 (1C, C-8), 105.0 (2C, C-2, C-6), 66.8 (4C, C-10, C-11), 60.9 (1C, $\left.\mathrm{OCH}_{3}\right), 56.1\left(2 \mathrm{C}, \mathrm{OCH}_{3}\right)$. HRMS-ESI: $\mathrm{m} / \mathrm{z}$ calcd. for $\mathrm{C}_{16} \mathrm{H}_{21} \mathrm{NO}_{5}(\mathrm{M}+\mathrm{H})^{+}$: 308.1492 found: 308.1294 .

\section{General procedure for the preparation of derivatives $\mathrm{N}$ -} hydroxysuccinimides $\mathbf{2 5 - 2 8}$

To a solution of $1.4 \mathrm{mmol}$ of $\mathbf{5 - 8}$ in $10 \mathrm{~mL}$ of dichloromethane were added $2.1 \mathrm{mmol}$ of $\mathrm{N}$-hydroxysuccinimide and $2.1 \mathrm{mmol}$ of pyridine. The mixture was stirred at room temperature until completion of the reaction, noticed by TLC. Then $2 \mathrm{M} \mathrm{HCl}$ was added to the mixture until $\mathrm{pH} 1$ and the product was extracted with ethyl acetate $(3 \times 25$ $\mathrm{mL})$. The organic layer was washed with $\mathrm{NaOH} 10 \%(3 \times$ $25 \mathrm{~mL}$ ), dried with anhydrous sodium sulfate, filtered and removed under reduced pressure affording the pure acyl derivatives after purified by recrystallization from ethanol/ water solution 80:20.

(E)-2,5-dioxopyrrolidin-1-yl cinnamate (25) This compound was prepared by $1.4 \mathrm{mmol}$ of $\mathbf{5}$ and $2.8 \mathrm{mmol}$ of $\mathrm{N}$ hydroxysuccinimide, according to the general procedure. The product was obtained in $83 \%$ yield as white crystals after purified by recrystallization from ethanol/water solution 80:20; $\mathrm{mp} 177-178^{\circ} \mathrm{C}$; IR $\left(\overline{\mathrm{U}} / \mathrm{cm}^{-1}\right)$ : 3065,2943 , $1755,1723,1625,1574 .{ }^{1} \mathrm{H}-\mathrm{NMR}\left(300 \mathrm{MHz}, \mathrm{CDCl}_{3}\right) \delta$ : $7.92\left(1 \mathrm{H}, \mathrm{d}\right.$, olefin, $\left.J^{3}=12.0 \mathrm{~Hz}\right), 7.58-7.25(5 \mathrm{H}, \mathrm{m}, \mathrm{Ar}-\mathrm{H})$, $6.68\left(1 \mathrm{H}, \mathrm{d}\right.$, olefin, $\left.J^{3}=12.0 \mathrm{~Hz}\right), 2.88(4 \mathrm{H}, \mathrm{s}$, methylenic). ${ }^{13} \mathrm{C}-\mathrm{NMR}\left(75 \mathrm{MHz}, \mathrm{CDCl}_{3}\right.$ ) $\delta: 169.2$ (1C, C-9), 162.0 (2C, C-10), 150.0 (1C, C-7), 133.4 (1C, C-1), 131.5 (1C, C-4), 129.0 (2C, C-3/C-5), 128.6 (2C, C-2/C-6), 111.5 (1C, C-8), 25.6 (2C, C-11). HRMS-ESI: $m / z$ calcd. for $\mathrm{C}_{13} \mathrm{H}_{11} \mathrm{NO}_{4}(\mathrm{M}$ $+\mathrm{Na})^{+}: 268.0580$ found: 268.0433 .

(E)-2,5-dioxopyrrolidin-1-yl 3-(4-methoxyphenyl)acrylate (26) This compound was prepared by $1.4 \mathrm{mmol}$ of 6 and $2.8 \mathrm{mmol}$ of $N$-hydroxysuccinimide, according to the general procedure. The product was obtained in $75 \%$ yield as white crystals after purified by recrystallization from ethanol/water solution 80:20; mp 148-149 ${ }^{\circ} \mathrm{C}$; IR $\left(\overline{\mathrm{U}} / \mathrm{cm}^{-1}\right)$ : 3061, 2937, 1757, 1725, 1623, 1595. ${ }^{1} \mathrm{H}-\mathrm{NMR}(300 \mathrm{MHz}$, $\left.\mathrm{CDCl}_{3}\right) \delta: 7.65\left(1 \mathrm{H}, \mathrm{d}\right.$, olefin, $\left.J^{3}=12.0 \mathrm{~Hz}\right), 7.51(2 \mathrm{H}, \mathrm{d}$, $\left.\mathrm{Ar}-\mathrm{H}, J^{3}=8,50 \mathrm{~Hz}\right), 6.91\left(2 \mathrm{H}, \mathrm{d}, \mathrm{Ar}-\mathrm{H}, J^{3}=8,50 \mathrm{~Hz}\right)$, $6.43\left(1 \mathrm{H}, \mathrm{d}\right.$, olefin, $\left.J^{3}=12.0 \mathrm{~Hz}\right), 3.84(3 \mathrm{H}, \mathrm{s}$, methylic $)$, $2.86\left(4 \mathrm{H}, \mathrm{s}\right.$, methylenic). ${ }^{13} \mathrm{C}-\mathrm{NMR}\left(75 \mathrm{MHz}, \mathrm{CDCl}_{3}\right) \delta$ : 169.4 (1C, C-9), 162.4 (2C, C-10), 162.3 (1C, C-4), 149.7 (1C, C-7), 130.5 (2C, C-2/C-6), 126.2 (1C, C-1), 114.5 (2C, $\mathrm{C}-3 / \mathrm{C}-5), 108.5$ (1C, C-8), $55.4\left(1 \mathrm{C}, \mathrm{OCH}_{3}\right), 25.5$ (2C, C11). HRMS-ESI: $\mathrm{m} / \mathrm{z}$ calcd. for $\mathrm{C}_{14} \overline{\mathrm{H}}_{13} \mathrm{NO}_{5}(\mathrm{M}+\mathrm{Na})^{+}$: 298.0686 found: 298.0526 .

(E)-2,5-dioxopyrrolidin-1-yl 3-(3,4-dimethoxyphenyl) acrylate (27) This compound was prepared by $1.4 \mathrm{mmol}$ of 7 and $2.8 \mathrm{mmol}$ of $N$-hydroxysuccinimide, according to the general procedure. The product was obtained in $88 \%$ yield as white crystals after purified by recrystallization from ethanol/ water solution $80: 20 ; \mathrm{mp} 149-150{ }^{\circ} \mathrm{C}$; IR $\left(\overline{\mathrm{U}} / \mathrm{cm}^{-1}\right)$ : 3074 , 2967, 1759, 1728, 1621, 1598. ${ }^{1} \mathrm{H}-\mathrm{NMR}\left(300 \mathrm{MHz}, \mathrm{CDCl}_{3}\right)$ $\delta: 7.84\left(1 \mathrm{H}, \mathrm{d}\right.$, olefin, $\left.J^{3}=12.0 \mathrm{~Hz}\right), 7.15\left(1 \mathrm{H}, \mathrm{d}, \mathrm{Ar}-\mathrm{H}, J^{3}=\right.$ $8,50 \mathrm{~Hz}), 7.06(1 \mathrm{H}, \mathrm{s}, \mathrm{Ar}-\mathrm{H}), 6.88\left(1 \mathrm{H}, \mathrm{d}, \mathrm{Ar}-\mathrm{H}, J^{3}=8.5\right.$ $\mathrm{Hz}), 6.43\left(1 \mathrm{H}, \mathrm{d}\right.$, olefin, $\left.J^{3}=12.0 \mathrm{~Hz}\right), 3.91(6 \mathrm{H}, \mathrm{s}$, methylic), $2.86\left(4 \mathrm{H}, \mathrm{s}\right.$, methylenic). ${ }^{13} \mathrm{C}-\mathrm{NMR}\left(75 \mathrm{MHz}, \mathrm{CDCl}_{3}\right) \delta$ : 169.4 (1C, C-9), 162.2 (2C, C-10), 152.2 (1C, C-5), 149.9 (1C, C-7), 149.3 (1C, C-4), 126.4 (1C, C-1), 123.8 (1C, C-2), 111.3 (1C, C-8), 109.8 (1C, C-3), 108.8 (1C, C-6), 55.9 (2C, $\left.\mathrm{OCH}_{3}\right), 25.6$ (2C, C-11). HRMS-ESI: $\mathrm{m} / \mathrm{z}$ calcd. for $\mathrm{C}_{15} \mathrm{H}_{15} \mathrm{NO}_{6}(\mathrm{M}+\mathrm{Na})^{+} 328.0792$ found: 328.0600 .

(E)-2,5-dioxopyrrolidin-1-yl 3-(3,4,5-trimethoxyphenyl) acrylate (28) This compound was prepared by $1.4 \mathrm{mmol}$ of 8 and $2.8 \mathrm{mmol}$ of $N$-hydroxysuccinimide, according to the general procedure. The product was obtained in $70 \%$ yield as white crystals after purified by recrystallization from aqueous ethanol $(80 \% \mathrm{v} / \mathrm{v}) ; \mathrm{mp} 176-177^{\circ} \mathrm{C}$; IR $\left(\overline{\mathrm{U}} / \mathrm{cm}^{-1}\right)$ : 3009, 2940, 1758, 1728, 1625, 1578. ${ }^{1} \mathrm{H}-\mathrm{NMR}(300 \mathrm{MHz}$, $\left.\mathrm{CDCl}_{3}\right) \delta: 7.82\left(1 \mathrm{H}, \mathrm{d}\right.$, olefin, $\left.J^{3}=12.0 \mathrm{~Hz}\right), 6.78(2 \mathrm{H}, \mathrm{s}$, Ar-H), $6.48\left(1 \mathrm{H}, \mathrm{d}\right.$, olefin, $\left.J^{3}=12.0 \mathrm{~Hz}\right), 3.89(9 \mathrm{H}, \mathrm{s}$, methylic), 2.86 (4H, s, methylenic). ${ }^{13} \mathrm{C}-\mathrm{NMR}(75 \mathrm{MHz}$, $\left.\mathrm{CDCl}_{3}\right) \delta: 169.3$ (1C, C-9), 162.0 (2C, C-10), 153.4 (2C, C3/C-5), 149.9 (1C, C-7), 141.2 (1C, C-4), 128.8 (1C, C-1), 110.5 (1C, C-8), 105.8 (2C, C-2/C-6), $60.9\left(1 \mathrm{C}, \mathrm{OCH}_{3}\right)$, $56.1\left(2 \mathrm{C}, \mathrm{OCH}_{3}\right), 25.6$ (2C, C-11). HRMS-ESI: $\mathrm{m} / z$ calcd. for $\mathrm{C}_{16} \mathrm{H}_{17} \mathrm{NO}_{7}(\mathrm{M}+\mathrm{Na})^{+}$: 358.0897 found: 358.0721 .

\section{In vitro bioassays}

\section{Antibacterial and antifungal activities evaluation}

The piplartine analogs were evaluated in vitro for their antimicrobial activities against the fungi through a Mueller Hinton broth microdilution method and with the methodology and interpretative criteria proposed by document M27A3 (CLSI 2008) and through a standard Mueller 
Scheme 1 Synthesis of piplartine analogs. i $\mathrm{SOCl}_{2}$, DMF, $\mathrm{CH}_{2} \mathrm{Cl}_{2}$, r.t.; ii $\mathrm{NH}_{2} \mathrm{NH}_{2}$. $\mathrm{H}_{2} \mathrm{O}, \mathrm{CH}_{3} \mathrm{CN}, 0^{\circ} \mathrm{C}$; iii phthalic anhydride, HAc, reflux; iv benzoyl chloride, piridine, $0{ }^{\circ} \mathrm{C}$; v morpholine, $\mathrm{CH}_{2} \mathrm{Cl}_{2}$, r.t.; vi

NHS, $\mathrm{CH}_{2} \mathrm{Cl}_{2}$, r.t

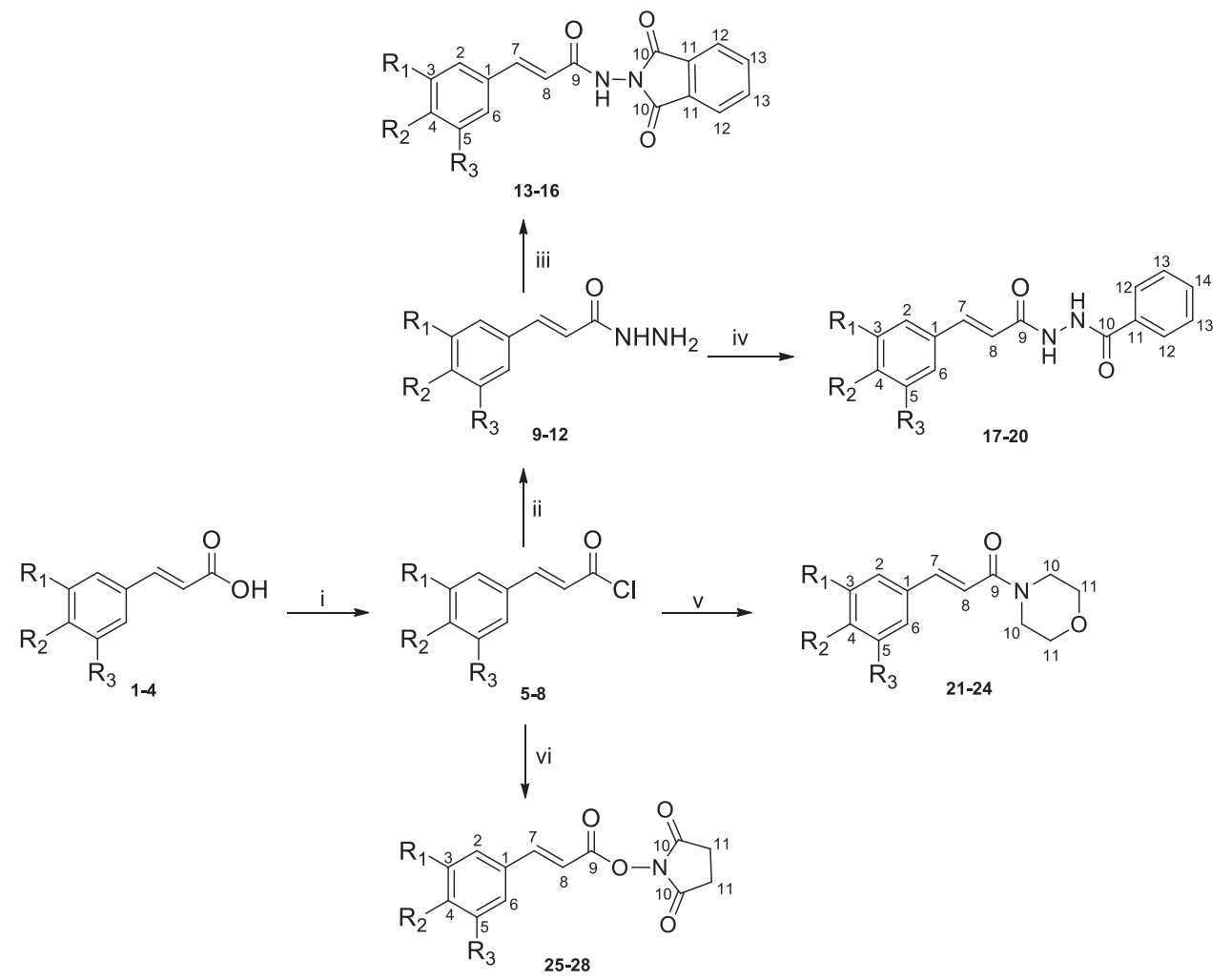

$\mathrm{mg} / \mathrm{mL}$ ) was added and the cells were incubated again for an additional $4 \mathrm{~h}$ period. Then, the medium was carefully removed and added to each well $100 \mu \mathrm{L}$ of DMSO. The plates were shaken for $5 \mathrm{~min}$ and absorbance for each sample was measured in a spectrophotometric microplate reader at $560 \mathrm{~nm}$. The percentage of cytotoxicity was calculated as $[(\mathrm{A}-\mathrm{B}) / \mathrm{A} \times 100)]$, where $\mathrm{A}$ and $\mathrm{B}$ are the absorbance of control and treated cells, respectively. Data were analyzed using linear regression to obtain values for $\mathrm{CC}_{50}$ and $\mathrm{CC}_{90}$ (cytotoxic concentration for 50 and $90 \%$ of cells, respectively). Selectivity indexes were expressed as the ratio $\mathrm{CC}_{50} / \mathrm{IC}_{50}$.

\section{Results and discussion}

\section{Chemistry}

The proposed piplartine analogs were obtained by a simple and objective synthetic route (Scheme 1); the compounds were achieved in good yields and high purity and characterized by infrared, ${ }^{1} \mathrm{H}$ and ${ }^{13} \mathrm{C}$ nuclear magnetic resonance and high resolution mass spectroscopy. Here in, several classes of compounds containing different groups have been synthesized in substitution for the heterocyclic ring of piplartin, such as $\alpha, \beta$-unsaturated carbonyl heterocyclic derivatives (phthalimides), $\alpha, \beta$-saturated carbonyl heterocyclic derivatives (succinimides), non-carbonylated 
Table 1 Chemical structures, yields and $\log \mathrm{P}$ values of the synthesized compounds<smiles>[Y]C(=O)/C=C/c1cc([R])c([R2])c([R])c1</smiles>

13-28

\begin{tabular}{|c|c|c|c|c|c|c|c|}
\hline Compound & $R_{1}$ & $R_{2}$ & $R_{3}$ & $X$ & $Y$ & $\operatorname{cLog} P^{\mathrm{a}}$ & Yield (\%) \\
\hline 13 & $\mathrm{H}$ & $\mathrm{H}$ & $\mathrm{H}$ & $\mathrm{NH}$ & & 2.45 & 90 \\
\hline 14 & $\mathrm{H}$ & $\mathrm{OCH}_{3}$ & $\mathrm{H}$ & $\mathrm{NH}$ & & 2.33 & 85 \\
\hline 15 & $\mathrm{OCH}_{3}$ & $\mathrm{OCH}_{3}$ & $\mathrm{H}$ & $\mathrm{NH}$ & & 2.2 & 70 \\
\hline 16 & $\mathrm{OCH}_{3}$ & $\mathrm{OCH}_{3}$ & $\mathrm{OCH}_{3}$ & $\mathrm{NH}$ & & 2.08 & 85 \\
\hline 17 & $\mathrm{H}$ & $\mathrm{H}$ & $\mathrm{H}$ & $\mathrm{NH}$ & & 2.64 & 94 \\
\hline 18 & $\mathrm{H}$ & $\mathrm{OCH}_{3}$ & $\mathrm{H}$ & $\mathrm{NH}$ & & 2.52 & 86 \\
\hline 19 & $\mathrm{OCH}_{3}$ & $\mathrm{OCH}_{3}$ & $\mathrm{H}$ & $\mathrm{NH}$ & & 2.39 & 82 \\
\hline 20 & $\mathrm{OCH}_{3}$ & $\mathrm{OCH}_{3}$ & $\mathrm{OCH}_{3}$ & $\mathrm{NH}$ & & 2.26 & 87 \\
\hline 21 & $\mathrm{H}$ & $\mathrm{H}$ & $\mathrm{H}$ & - & & 1.35 & 72 \\
\hline 22 & $\mathrm{H}$ & $\mathrm{OCH}_{3}$ & $\mathrm{H}$ & - & & 1.23 & 75 \\
\hline 23 & $\mathrm{OCH}_{3}$ & $\mathrm{OCH}_{3}$ & $\mathrm{H}$ & - & & 1.1 & 72 \\
\hline 24 & $\mathrm{OCH}_{3}$ & $\mathrm{OCH}_{3}$ & $\mathrm{OCH}_{3}$ & - & & 0.97 & 86 \\
\hline 25 & $\mathrm{H}$ & $\mathrm{H}$ & $\mathrm{H}$ & $\mathrm{O}$ & & 2.1 & 83 \\
\hline
\end{tabular}


Table 1 continued<smiles>[Y]C(=O)C=Cc1cc([R])c([R])c([R])c1</smiles>

13-28

\begin{tabular}{|c|c|c|c|c|c|c|c|}
\hline Compound & $R_{1}$ & $R_{2}$ & $R_{3}$ & $X$ & $Y$ & $\operatorname{cLog} P^{\mathrm{a}}$ & Yield (\%) \\
\hline 26 & $\mathrm{H}$ & $\mathrm{OCH}_{3}$ & $\mathrm{H}$ & $\mathrm{O}$ & & 2.0 & 75 \\
\hline 27 & $\mathrm{OCH}_{3}$ & $\mathrm{OCH}_{3}$ & $\mathrm{H}$ & $\mathrm{O}$ & & 1.7 & 88 \\
\hline 28 & $\mathrm{OCH}_{3}$ & $\mathrm{OCH}_{3}$ & $\mathrm{OCH}_{3}$ & $\mathrm{O}$ & & 1.4 & 70 \\
\hline Piplartine & $\mathrm{OCH}_{3}$ & $\mathrm{OCH}_{3}$ & $\mathrm{OCH}_{3}$ & - & & 1.6 & - \\
\hline
\end{tabular}

${ }^{\mathrm{a}}$ The $\log P$ values were determined by ChemBioDraw Ultra 12.0 program

heterocyclic derivatives (amides) and finally nonheterocyclic compounds (acylhydrazides). With these chemical variations combined with the variations of the trimethoxylated ring of piplartine, preliminary information about structure-antimicrobial activity relationship could be determined.

The initial step of the synthetic route involved the synthesis of acyl chlorides (5-8) from four commercial cinnamic acids (cinnamic acid, 4-methoxycinnamic acid, 3,4-dimethoxycinnamic acid and 3,4,5-trimethoxycinnamic acid) (Carvalho et al. 2012). The crude dichloromethane solution of these acyl chlorides was employed in the synthesis of hydrazides 9-12 which were also used without prior purification in the following steps. Phthalimides 13-16 were obtained in yields higher than $70 \%$ from the reaction of hydrazides 9-12 with phthalic anhydride in glacial acetic acid under heating (Joshi et al. 2014). The analysis of ${ }^{13} \mathrm{C}-\mathrm{NMR}$ spectra of phthalimides showed two signals corresponding to the carbonyl carbons of the compounds between 166.1-164.6 ppm, confirming the cyclization reaction and formation of phthalimide derivatives. The IR spectra of these compounds also showed bands corresponding to carbonyl groups between 1737 and $1791 \mathrm{~cm}^{-1}$. The singlet corresponding to the NH proton was observed between 11 and $10 \mathrm{ppm}$ in ${ }^{1} \mathrm{H}-\mathrm{NMR}$ spectra. The reaction of hydrazides with benzoyl chloride provided the benzoyl derivatives $\mathbf{1 7 - 2 0}$ in yields higher than $82 \%$. In the ${ }^{1} \mathrm{H}-\mathrm{NMR}$ spectra, it was possible to observe a coupling constant $\left(J^{3}\right)$ of $1.5 \mathrm{~Hz}$ for the two NH protons and these doublets were registered near 10.5 and $10.2 \mathrm{ppm}$. Two signals corresponding to the two carbonyl groups were observed between 165.7-164.4 ppm, confirming the successful benzoylation of hydrazides. The amides $\mathbf{2 1 - 2 4}$ were smoothly synthesized by the reaction of acyl chlorides 5-8 with morpholine and were obtained in high yields $(>72 \%)$. It was possible to notice a broad signal at $3.7 \mathrm{ppm}$ relative to the eight methylene protons of the morpholine moiety in amides 21-24 in their ${ }^{1} \mathrm{H}$ NMR spectra and one signal near $165 \mathrm{ppm}$ corresponding to the amidic carbonyl in their ${ }^{13} \mathrm{C}-\mathrm{NMR}$ spectra. The bands referring to the amidic carbonyl were recorded near 1640 $\mathrm{cm}^{-1}$ in their infrared spectra. The reaction of acyl chlorides 5-8 with $N$-hydroxysuccinimide afforded the pure acylated derivatives 25-28 in yields higher than 70\% after recrystallization from ethanol/water. In their ${ }^{1} \mathrm{H}-\mathrm{NMR}$ spectra, it was possible to observe a singlet at $2.8 \mathrm{ppm}$ relative to the four methylene protons and two signals between 169.4-162.0 ppm in their ${ }^{13} \mathrm{C}$-NMR spectra, corresponding to the carbonyl groups of the compounds. Finally, it is important to note that the protons related to the cinnamic subunit olefin have been preserved $\left(J^{3}=12 \mathrm{~Hz}\right)$, which proves the absence of 
conjugate addition products and the maintenance of $E$ configuration. (Table 1).

\section{Antimicrobial and toxic assays}

The toxicity action of piplartine and its analogs and reference drugs on healthy hamster kidney cells (BHK-21) was evaluated and the results are shown in Table 2 . This evaluation was necessary to establish the indices of selectivity with respect to antifungal and antibacterial actions.

Piplartine and its analogs were evaluated against Candida albicans, C. tropicalis, C. krusei, C. glabrata and $C$. parapsilosis; the results of the investigation on these yeasts are reported here for the first time, along with their selectivity indexes (Table 3).

Fluconazole was used as a reference fungistatic drug and the results were estimated as the minimum concentration required to inhibit $50 \%$ ( $\mathrm{IC}_{50}$, related to fungistatic activity) and $90 \%$ ( $\mathrm{IC}_{90}$, related to fungicidal activity) of fungal cell growth. Piplartine presented the broadest spectrum of antifungal activity, and was shown to be fungistatic against all fungi stains evaluated, except $C$. tropicalis, in the range of 94.60-189.20 $\mu \mathrm{M}$. However, piplartine was the most toxic compound to BHK-21 cell culture with the lowest selective index range, between 0.2 and 0.4 . This high toxicity prevents the substances for future in vivo testing.

Table 2 In vitro cytotoxicity $(\mu \mathrm{M})$ against hamster kidney cell culture (BHK-21)

\begin{tabular}{llr}
\hline Compound & $\mathrm{CC}_{50}$ & $\mathrm{CC}_{90}$ \\
\hline $\mathbf{1 3}$ & 285.12 & 452.13 \\
$\mathbf{1 4}$ & 252.91 & 396.65 \\
$\mathbf{1 5}$ & 239.98 & 379.21 \\
$\mathbf{1 6}$ & 156.73 & 427.23 \\
$\mathbf{1 7}$ & 231.71 & 439.04 \\
$\mathbf{1 8}$ & 212.75 & 402.75 \\
$\mathbf{1 9}$ & 188.18 & 361.64 \\
$\mathbf{2 0}$ & 125.20 & 333.19 \\
$\mathbf{2 1}$ & 354.75 & 586.79 \\
$\mathbf{2 2}$ & 295.28 & 506.59 \\
$\mathbf{2 3}$ & 281.96 & 458.91 \\
$\mathbf{2 4}$ & 185,55 & 384.38 \\
$\mathbf{2 5}$ & 259.93 & 462.70 \\
$\mathbf{2 6}$ & 249.93 & 429.70 \\
$\mathbf{2 7}$ & 196.34 & 397.76 \\
$\mathbf{2 8}$ & 174.99 & 415.13 \\
Piplartine & 40.14 & 80.31 \\
Fluconazole & 382.60 & 521.13 \\
Chloramphenicol & 269.18 & 486.52 \\
\hline
\end{tabular}

Among the synthesized piplartine analogs, morpholine 3,4,5-trimethoxylated derivative $\mathbf{2 4}$ was the most promising analog showing activity against $C$. krusei at $48.83 \mu \mathrm{M}$, and a selectivity index greater than three. This compound was almost four-fold more potent and about five-fold less toxic than piplartine $\left(\mathrm{IC}_{50}: 189.2 \mu \mathrm{M}\right.$; selectivity index of 0.21 ). Furthermore, derivative $\mathbf{2 4}$ was more than two-fold more effective than fluconazole $\left(\mathrm{IC}_{50}: 104.48 \mu \mathrm{M}\right)$ against this strain. Although less active than fluconazole, derivative $\mathbf{2 4}$ also showed activity against $C$. tropicalis at $97.67 \mu \mathrm{M}$, while piplartine was inactive up to the highest evaluated concentration. It is also worth noting that piperazine or morpholine bioisosteric rings play a crucial role in antimicrobial activity, as in drugs such as linezolid, eperezolid and itraconazole or in morpholine-eugenol derivatives reported in our recent paper (26). Moreover, it is important to note that derivative $\mathbf{2 4}$ showed the lowest $\log \mathrm{P}$ value (0.97) among all the synthesized compounds, suggesting an interesting value for anti-Candida activity (Table 1).

Eleven other analogs of piplartine were moderately active against different Candida species. Phthalimide derivatives 13, 14, and 15 showed some activity against $C$. tropicalis; for these three compounds, there is a clear and direct correlation between the number of methoxyl substituents on the aromatic ring and the activity against this strain $\left(\mathrm{IC}_{50}\right.$ of 13 : $342.32 \mu \mathrm{M}$; $\mathrm{IC}_{50}$ of $14: 310.47 \mu \mathrm{M} ; \mathrm{IC}_{50}$ of 15: $170.40 \mu \mathrm{M}$ ), which may suggest that the investigation of other ring substitution patterns may lead to the discovery of more active antifungal agents. Again, it can be seen that a lower $\log \mathrm{P}$ value is related to a better antifungal activities, since derivative 15 has a $\log \mathrm{P} 2.2$. On the other hand, only the phthalimide derivative 16 (3,4,5-trimethoxy-substituted) showed some activity against $C$. krusei and it was not active against $C$. tropicalis, suggesting that more than two methoxyl groups at this aromatic ring is unfavorable for activity against this species.

Among the benzoylhydrazides (17-20), derivative 17 showed a broader spectrum of action, presenting activity against all species evaluated, except $C$. krusei, although at a low potency $\left(\mathrm{IC}_{50}: 375.7 \mu \mathrm{M}\right)$. Derivative 19 was the most potent of the four acylhydrazides, showing $\mathrm{IC}_{50}$ of 183.9 $\mu \mathrm{M}$ against $C$. glabrata, and once again it was noticed that a 3,4-disubstituted cinnamic ring may be related to a better antifungal activity. Tri-substituted acylhydrazide $\mathbf{2 0}$ was also moderately active at $280.7 \mu \mathrm{M}$ against $C$. albicans, $C$. tropicalis, and C. krusei.

It was also observed that the increasing in the number of methoxyl groups in the cinnamic ring of the $O$-acyl-succinic derivatives and the decreasing of their $\log \mathrm{P}$ values led to an increase in activity against $C$. tropicalis, as noted for the phthalic derivatives. Succinic unsubstituted derivative 25 $(\log \mathrm{P} 2.1)$ was inactive against $C$. tropicalis, while the three-substituted (26; $\log \mathrm{P}$ 2.0), 3,4-disubstituted (27; $\log \mathrm{P}$ 
Table 3 In vitro antifungal activity $(\mu \mathrm{M})$ and selectivity index (SI) of piplartine and synthesized analogs

\begin{tabular}{|c|c|c|c|c|c|c|}
\hline Compound & & $\begin{array}{l}\text { C. albicans } \\
\text { ATCC } 10231 \\
\text { (SI) }\end{array}$ & $\begin{array}{l}\text { C. tropicalis } \\
\text { ATCC } 750 \text { (SI) }\end{array}$ & $\begin{array}{l}\text { C. krusei } \\
\text { ATCC } 6258 \\
\text { (SI) }\end{array}$ & $\begin{array}{l}\text { C. glabrata } \\
\text { ATCC } 90030 \\
\text { (SI) }\end{array}$ & $\begin{array}{l}\text { C. parapsilosis } \\
\text { ATCC } 22019 \\
\text { (SI) }\end{array}$ \\
\hline \multirow[t]{2}{*}{13} & $\mathrm{IC}_{50}$ & $-^{\mathrm{a}}$ & $342.32(0.83)$ & - & - & - \\
\hline & $\mathrm{IC}_{90}$ & - & - & - & - & - \\
\hline \multirow[t]{2}{*}{14} & $\mathrm{IC}_{50}$ & - & $310.47(0.81)$ & - & - & - \\
\hline & $\mathrm{IC}_{90}$ & - & - & - & - & - \\
\hline \multirow[t]{2}{*}{15} & $\mathrm{IC}_{50}$ & - & $170.40(1.4)$ & - & - & - \\
\hline & $\mathrm{IC}_{90}$ & - & - & - & - & - \\
\hline \multirow[t]{2}{*}{16} & $\mathrm{IC}_{50}$ & - & - & $261.70(0.59)$ & - & - \\
\hline & $\mathrm{IC}_{90}$ & - & - & - & - & - \\
\hline \multirow[t]{2}{*}{17} & $\mathrm{IC}_{50}$ & $375.79(0.61)$ & $375.79(0.61)$ & - & $375.79(0.61)$ & $375.79(0.61)$ \\
\hline & $\mathrm{IC}_{90}$ & - & - & - & - & - \\
\hline \multirow[t]{2}{*}{18} & $\mathrm{IC}_{50}$ & - & - & - & - & - \\
\hline & $\mathrm{IC}_{90}$ & - & - & - & - & - \\
\hline \multirow[t]{2}{*}{19} & $\mathrm{IC}_{50}$ & - & - & - & $183.98(1.02)$ & - \\
\hline & $\mathrm{IC}_{90}$ & - & - & - & - & - \\
\hline \multirow[t]{2}{*}{20} & $\mathrm{IC}_{50}$ & $280.79(0.44)$ & $280.79(0.44)$ & $280.79(0.44)$ & - & - \\
\hline & $\mathrm{IC}_{90}$ & - & - & - & $280.79(1.18)$ & $280.79(1.18)$ \\
\hline \multirow[t]{2}{*}{21} & $\mathrm{IC}_{50}$ & - & $460.59(0.77)$ & $460.59(0,77)$ & - & $460.59(0.77)$ \\
\hline & $\mathrm{IC}_{90}$ & - & - & - & - & - \\
\hline \multirow[t]{2}{*}{22} & $\mathrm{IC}_{50}$ & - & - & - & - & - \\
\hline & $\mathrm{IC}_{90}$ & - & - & - & - & - \\
\hline \multirow[t]{2}{*}{23} & $\mathrm{IC}_{50}$ & - & - & - & - & - \\
\hline & $\mathrm{IC}_{90}$ & - & - & - & - & - \\
\hline \multirow[t]{2}{*}{24} & $\mathrm{IC}_{50}$ & - & $97.67(1.89)$ & $48.83(3.79)$ & - & - \\
\hline & $\mathrm{IC}_{90}$ & - & - & - & - & - \\
\hline \multirow[t]{2}{*}{25} & $\mathrm{IC}_{50}$ & - & - & - & - & - \\
\hline & $\mathrm{IC}_{90}$ & - & - & - & - & - \\
\hline \multirow[t]{2}{*}{26} & $\mathrm{IC}_{50}$ & - & $218.12(1.14)$ & $363.54(0.68)$ & - & - \\
\hline & $\mathrm{IC}_{90}$ & - & - & - & - & - \\
\hline \multirow[t]{2}{*}{27} & $\mathrm{IC}_{50}$ & - & $196.66(0.99)$ & - & - & - \\
\hline & $\mathrm{IC}_{90}$ & - & - & - & - & - \\
\hline \multirow[t]{2}{*}{28} & $\mathrm{IC}_{50}$ & - & $179.05(0.97)$ & - & - & - \\
\hline & $\mathrm{IC}_{90}$ & - & - & - & - & - \\
\hline \multirow[t]{2}{*}{ Pip. } & $\mathrm{IC}_{50}$ & $94.60(0.42)$ & - & $189.20(0.21)$ & $189.20(0.21)$ & $94.60(0.42)$ \\
\hline & $\mathrm{IC}_{90}$ & - & - & - & - & - \\
\hline Fluc. & $\mathrm{IC}_{50}$ & $1.63(234)$ & $3.26(117)$ & $104.48(3.6)$ & $52.24(7.3)$ & $3.26(117)$ \\
\hline
\end{tabular}

Pip. Piplartine, Fluc. Fluconazole

${ }^{a}$ Inactive at highest evaluated concentration
1.7) and 3,4,5-trisubstituted (28, $\log \mathrm{P} 1.4)$ derivatives were active at 218.12, 196.66, and $179.05 \mu \mathrm{M}$, respectively, against this strain.

Piplartine, as well as its synthetic analogs, were also assessed for their antibacterial potential against Staphylococcus aureus, Eschericha coli and Pseudomonas aeruginosa strains using the same concentrations and dilutions employed in the antifungal assays.
The results and selectivity indexes on BHK-21 cells are shown in Table 4. Three piplartine analogs were active against the same strain and it was noticed that all of these active derivatives bear an unsubstituted cinnamic ring (13, 17 and 25 compounds). Among them, benzyl hydrazide 17 was more than three-fold more potent than the prototype piplartine, showing an $\mathrm{IC}_{50}$ value of $85.20 \mu \mathrm{M}$ and a greater selectivity index (2.71). This suggests that new 
Table 4 In vitro antibacterial activity $(\mu \mathrm{M})$ and selectivity index (SI) of piplartine and synthesized analogs

\begin{tabular}{|c|c|c|c|c|}
\hline Compound & & $\begin{array}{l}\text { S. aureus } \\
\text { ATCC } 6538 \text { (SI) }\end{array}$ & $\begin{array}{l}\text { E. coli } \\
\text { ATCC } 25922 \\
\text { (SI) }\end{array}$ & $\begin{array}{l}\text { P. aeruginosa } \\
\text { ATCC } 9027 \\
\text { (SI) }\end{array}$ \\
\hline \multirow[t]{2}{*}{13} & $\mathrm{IC}_{50}$ & $205.42(1.38)$ & $--^{\mathrm{a}}$ & - \\
\hline & $\mathrm{IC}_{90}$ & - & - & - \\
\hline \multirow[t]{2}{*}{14} & $\mathrm{IC}_{50}$ & - & - & - \\
\hline & $\mathrm{IC}_{90}$ & - & - & - \\
\hline \multirow[t]{2}{*}{15} & $\mathrm{IC}_{50}$ & - & - & - \\
\hline & $\mathrm{IC}_{90}$ & - & - & - \\
\hline \multirow[t]{2}{*}{16} & $\mathrm{IC}_{50}$ & - & - & - \\
\hline & $\mathrm{IC}_{90}$ & - & - & - \\
\hline \multirow[t]{2}{*}{17} & $\mathrm{IC}_{50}$ & $85.20(2.71)$ & - & - \\
\hline & $\mathrm{IC}_{90}$ & - & - & - \\
\hline \multirow[t]{2}{*}{18} & $\mathrm{IC}_{50}$ & - & - & - \\
\hline & $\mathrm{IC}_{90}$ & - & - & - \\
\hline \multirow[t]{2}{*}{19} & $\mathrm{IC}_{50}$ & - & - & - \\
\hline & $\mathrm{IC}_{90}$ & - & - & - \\
\hline \multirow[t]{2}{*}{20} & $\mathrm{IC}_{50}$ & - & - & - \\
\hline & $\mathrm{IC}_{90}$ & - & - & - \\
\hline \multirow[t]{2}{*}{21} & $\mathrm{IC}_{50}$ & - & - & - \\
\hline & $\mathrm{IC}_{90}$ & - & - & - \\
\hline \multirow[t]{2}{*}{22} & $\mathrm{IC}_{50}$ & - & - & - \\
\hline & $\mathrm{IC}_{90}$ & - & - & - \\
\hline \multirow[t]{2}{*}{23} & $\mathrm{IC}_{50}$ & - & - & - \\
\hline & $\mathrm{IC}_{90}$ & - & - & - \\
\hline \multirow[t]{2}{*}{24} & $\mathrm{IC}_{50}$ & - & - & - \\
\hline & $\mathrm{IC}_{90}$ & - & - & - \\
\hline \multirow[t]{2}{*}{25} & $\mathrm{IC}_{50}$ & $408.06(0.63)$ & - & - \\
\hline & $\mathrm{IC}_{90}$ & - & - & - \\
\hline \multirow[t]{2}{*}{26} & $\mathrm{IC}_{50}$ & - & - & - \\
\hline & $\mathrm{IC}_{90}$ & - & - & - \\
\hline \multirow[t]{2}{*}{27} & $\mathrm{IC}_{50}$ & - & - & - \\
\hline & $\mathrm{IC}_{90}$ & - & - & - \\
\hline \multirow[t]{2}{*}{28} & $\mathrm{IC}_{50}$ & - & - & - \\
\hline & $\mathrm{IC}_{90}$ & - & - & - \\
\hline \multirow[t]{2}{*}{ Pip. } & $\mathrm{IC}_{50}$ & $315.33(0.12)$ & - & - \\
\hline & $\mathrm{IC}_{90}$ & - & - & - \\
\hline Chl. & $\mathrm{IC}_{50}$ & $0.975(276)$ & $0.975(276)$ & 16.5 \\
\hline
\end{tabular}

Pip. Piplartine, Chl. Choramphenicol

${ }^{a}$ Inactive at highest evaluated concentration acylhydrazides can be exploited as interesting potential antibacterial agents. Phthalic derivative $\mathbf{1 3}$ was moderately active at $205 \mu \mathrm{M}$ (selectivity index of 1.38) and the succinic derivative 25 at $408.06 \mu \mathrm{M}$ (0.63 selectivity index). All of the compounds were inactive against Gram-negative $E$. coli or $P$. aeruginosa strains up to the highest concentration employed; however, piplartine and some synthetic analogs showed potential against Gram-positive S. aureus. Piplartine was the least active and the most toxic compound against this strain, showing $\mathrm{IC}_{50}$ value of $315.33 \mu \mathrm{M}$ and a low selectivity index of 0.12 . Again, synthetic analogs showed less toxicity than piplartine. Different from what was noted in the results of antifungal assessment, higher $\log P$ values contributed to better antibacterial activity of the compounds in each synthesized series (Tables 1 and 4).

Based on the results found in antimicrobial tests it was possible to realize the major structural requirements for antifungal and antibacterial activities among the synthesized piplartine analogs (Fig. 2).

\section{Conclusion}

Sixteen piplartine analogs were synthesized and these compounds were assessed for their antimicrobial potential and toxicity. Derivative $\mathbf{2 4}$ showed the best results against C. krusei (more than twice as potent as fluconazole, fourfold more potent and five-fold less toxic than piplartine). Other analogs had moderate activity against different fungal strains and a direct correlation was seen between the number of aromatic methoxyl groups and antifungal activity. Furthermore, benzoyl hydrazide $\mathbf{1 7}$ was more than three-fold more potent than piplartine in antibacterial evaluation against $S$. aureus and five-fold less toxic than piplartine. It was possible to note that an aromatic ring lacking methoxyl groups is important for the antibacterial activity of these compounds. These findings encourage the design and synthesis of new piplartine analogs as potential and safer candidates to new antimicrobial agents.
Fig. 2 Structure-antimicrobial activity relationships for piplartine analogs

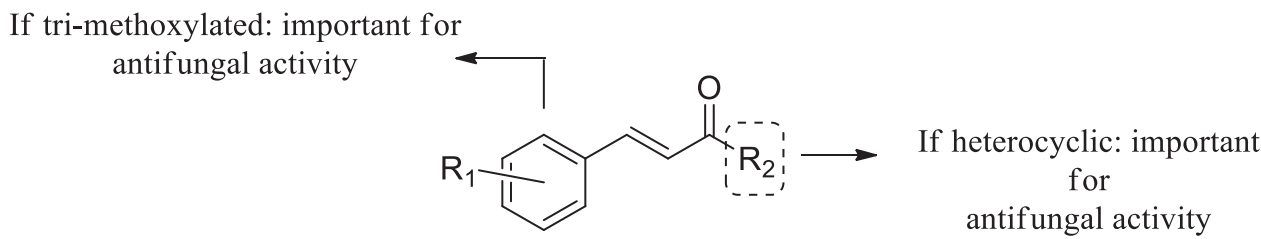

If non-substituted: important for antibacterial activity 
Acknowledgments This work was supported by FAPEMIG (APQ01209-13), CAPES, CNPq and FINEP.

\section{Compliance with Ethical Standards}

Conflict of Interest The authors declare that they have no competing interests.

\section{References}

Abrão PHO, Pizi RB, Souza TB, Silva NC, Fregnan AM, Silva FN, Coelho LFL, Malaquias LCC, Dias ALT, Dias DF, Veloso MP, Carvalho DT (2015) Synthesis and biological evaluation of new eugenol Mannich bases as promising antifungal agents. Chem Biol Drug Des 86:459-465

Adams DJ, Dai M, Pellegrino G, Wagner BK, Stern AM, Shamji AF, Schreiber SL (2012) Synthesis, cellular evaluation, and mechanism of action of piperlongumine analogs. PNAS 109:15115-15120

Alécio AC, Bolzani VS, Young MCM, Kato MJ, Furlan M (1998) Antifungal amide from leaves of Piper hispidum. J Nat Prod 61:637-639

Balunas MJ, Kinglorn AD (2005) Drug discovery from medicinal plants. Life Sci 78:431-441

Bezerra DP, Castro FO, APNN Alves, Pessoa C, Moraes MO, Silveira ER, Lima MAS, Elmino FJM, Costa-Lotufo LV (2006) In vivo growth-inhibition of Sarcoma 180 by piplartinee and piperine, two alkaloid amides from Piper. Braz J Med Biol Res 39:801-807

Bezerra DP, Pessoa C, Moraes MO, Saker-Neto N, Silveira ER, CostaLotufo LV (2013) Overview of the therapeutic potential of Piplartine (piperlongumine). Eur J Pharm Sci 48:453-463

Carvalho SA, Feitosa LO, Soares M, Costa TEMM, Henriques MG, Salomão K, Castro SL, Kaiser M, Brum R, Wardell JL, Wardell SMSV, Trossini GHG, Andricopulo AD, Barreiro EB, Mansour CAM (2012) Design and synthesis of new (E)-cinnamic $N$-acylhydrazones as potent antitrypanosomal agents. Eur J Med Chem 54:512-521

Chen J, Li W, Yao H, Xu J (2015) Insights into drug discovery from natural products through structural modification. Fitoterapia 103:231-241

Chin Y, Balunas MJ, Chai H, Kinghorn AD (2006) Drug discovery from natural sources. AAPS J 8:239-253

Cotinguiba F, Regasini LO, Bolzani VS, Debonsi HM, Passerini GD, Cicarelli RMD, Kato MJ, Furlan M (2009) Piperamides and their derivatives as potential anti-trypanosomal agents. Med Chem Res 18:703-711

Cragg MG, Newman J (2005) Plants as a source of anti-cancer agents. J. Ethnopharmacol 100:72-79

Clinical And Laboratory Standards Institute CLSI (2008) Reference method for broth dilution antifungal susceptibility testing of yeasts; Approved standard M27-A3, 3th edn. National Committee for Clinical Laboratory Standards, Wayne, PA

Clinical And Laboratory Standards Institute CLSI (2012) Methods for dilution antimicrobial susceptibility testes for bacteria that grow aerobically; Approved standard M07-A9, 9th edn. National Committee for Clinical Laboratory Standards, Wayne, PA

Joshi SD, More U, Dixit SR, Korat HH, Aminabhavi TM, Badiger AM (2014) Synthesis, characterization, biological activity, and 3DQSAR studies on some novel class of pyrrole derivatives as antitubercular agents. Med Chem Res 23:1123-1147

Kumar JU, Shankaraiah G, Kumar RSC, Pitke VV, Rao GT, Poornima B, Babu KS, Sreedhar AS (2013) Synthesis, anticancer, and antibacterial activities of piplartine derivatives on cell cycle regulation and growth inhibition. J Asian Nat Prod Res 15:658-669
Lampe JW (2003) Spicing up a vegetarian diet: chemopreventive effects of phytochemicals. Am J Clin Nutr 78:579-583

Lee SE, Park BS, Bayman P, Baker JL, Choi WS, Campbell BC (2007) Campbell, suppression of ochratoxin biosynthesis by naturally occurring alkaloids. Food Add Contam 24:391-397

Lee S, Park B, Kim M, Choi W, Kim H, Cho K, Lee S, Lee H (2001) Fungicidal activity of pipernonaline, a piperidine alkaloid derived from long pepper, Piper longum L., against phytopathogenic fungi. Crop Prot 20:523-528

Ling LL, Scheider T, Peoples AJ, Spoering AL, Engels I, Conlon BP, Mueller A, Schäberle TF, Hughes DE, Epstein S, Jones M, Lazarides L, Steadman VA, Cohen DR, Feliz CR, Fetterman KA, Mellert WP, Nitti AG, Zullo AM, Chen C, Lewis K (2015) A new antibiotic kills pathogens without detectable resistance. Nature 517:455-459

Low CY, Rotstein C (2011) Emerging fungal infections in immunocompromised patients. F100 Med Rep 3:1-8

Moraes J, Nascimento C, Yamaguchi LF, Kato MJ, Nakano E (2012) Schistosoma mansoni: In vitro schistosomicidal activity and tegumental alterations induced by piplartine on schistosomula. Exp Parasitol 132:222-227

Naika R, Prasannal KP, Ganapathy PSS (2010) Antibacterial activity of piperlongumine an alkaloid isolated from methanolic root extract of Piper longum L. Pharmacophore 1:141-148

Navickiene HMD, Alécio AC, Kato MJ, Bolzani VS, Young MCM, Cavalheiro AJ, Furlan M (2006) Antifungal amides from Piper hispidum and Piper tuberculatum. Phytochemistry 55:621-626

Okwute SK, Egharevba HO (2013) Egharevba, Piperine-type amides: review of the chemical and biological characteristics. Int J Chem 5:99-122

Park B, Son D, Choi W, Takeoka GR, Han SO, Kim T, Lee S (2008) Antiplatelet activities of newly synthesized derivatives of piperlongumine. Phytother Res 22:1195-1199

Paterson I, Anderson EA (2005) The renaissance of natural products as drug candidates. Science 310:451-453

Prashanth MK, Revanasiddappa HD, Rai KML, Veeresh B (2012) Synthesis, characterization, antidepressant and antioxidant activity of novel piperamides bearing piperidine and piperazine analogues. Bioorg Med Chem Lett 22:7065-7070

Prithwiraj GKY, Constant P, Bedos-Belval F, Duran H, Saffon N, Daffe M, Baltas M (2011) Design, synthesis, and biological evaluation of new cinnamic derivatives as antituberculosis agents. J Med Chem 54:1449-1461

Rao VR, Muthenna P, Shankaraiah G, Akileshwari C, Babu KH, Suresh G, Babu KS, Kumar RSC, Prasad KR, Yadav PA, Petrash JM, Reddy GB, Rao JM (2012) Synthesis and biological evaluation of new piplartine analogues as potent aldose reductase inhibitors (ARIs). Eur J Med Chem 57:344-361

Reddy PS, Jamil K, Madhusudhan P, Anjani G, Das B (2011) Antibacterial activity of isolates from Piper longum and Taxus baccata. Pharm Biol 39:36-238

Silva RV, Navickiene HMD, Kato MJ, Bolzani VS, Méda CI, Young MCM, Furlan M (2012) Antifungal amides from Piper arboreum and Piper tuberculatum. Phytochemistry 59:521-527

Souza TB, Orlandi M, Coelho LFL, Malaquias LCC, Dias ALT, Carvalho RR, Silva NC, Carvalho DT (2014) Synthesis and in vitro evaluation of antifungal and cytotoxic activities of eugenol glycosides. Med Chem Res 23:496-502

Souza TB, Raimundo POB, Andrade SA, Hipólito TMM, Silva NCS, Dias ALT, Ikegaki M, Rocha RP, Coelho LFL, Veloso MP, Carvalho DT, Dias DF (2015) Synthesis and antimicrobial activity of 6-triazolo-6-deoxy eugenol glucosides. Carbohydr Res 410:1-8

Sun L, Wang F, Dai F, Wang Y, Lin D, Zhou B (2015) Development and mechanism investigation of a new piperlongumine derivative as a potent anti-inflammatory agent. Biochem Pharmacol 95:156-169 